Untersuchung des Magdeburger Leitungswassers.

Von Dr. OTto Wendel.

Chem. Laboratorium Dr. Hugo Schulz, Magdeburg.

(Eingeg. 24.j1. 1916.)

Seit einer längeren Reihe von Jahren habe ich, beginnend vom Jahre 1904, in dieser Zeitschrift ${ }^{1}$ ) sowie in den Sonntagsnummern der Magdeburgischen Zeitung regelmäßig Untersuchungen des Magdeburger Elb- und Leitungswassers veröffentlicht.

Nachstehend gebe ich Zusammenstellungen und Durchschnittsberechnungen der Untersuchungen des Jahres 1915. Tabelle I.

\begin{tabular}{|c|c|c|c|c|c|c|c|c|c|}
\hline & 要 & & $\begin{array}{l}\text { Chew } \\
000000\end{array}$ & $\begin{array}{l}\text { aische } \\
\text { Teilen }\end{array}$ & $\begin{array}{l}\text { Untersu } \\
\text { filtriert }\end{array}$ & $\begin{array}{l}\text { chung } \\
\text { en Wa }\end{array}$ & issers & & \\
\hline $\begin{array}{c}\text { (Probenahmestelle } \\
\text { SteinstraBe 7) } \\
\text { vom Jahre } 1915\end{array}$ & 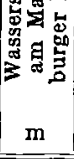 & 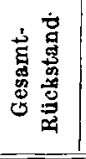 & 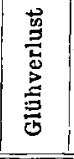 & 容 & 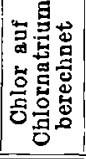 & 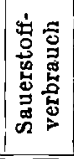 & 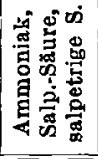 & 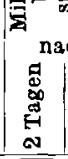 & 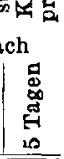 \\
\hline & $(+)$ & & & & & & & & \\
\hline $\begin{array}{l}\text { 4. Januar } \\
11 . \text { " }\end{array}$ & $\begin{array}{l}1,30 \\
2,52\end{array}$ & $\begin{array}{l}26,00 \\
28,20\end{array}$ & $\left|\begin{array}{l}3,60 \\
4,00\end{array}\right|$ & $\begin{array}{l}6,38 \\
6,03\end{array}$ & $\begin{array}{r}10,52 \\
9,94\end{array}$ & $\begin{array}{l}0,46 \\
0,45\end{array}$ & $\begin{array}{c}\text { feblen } \\
"\end{array}$ & $\begin{array}{l}19 \\
28\end{array}$ & $\begin{array}{l}45 \\
97\end{array}$ \\
\hline 18. & 3,20 & 22,00 & 4,00 & 3,55 & 5,85 & 0,46 & $n$ & 53 & 161 \\
\hline 25. & 3,18 & 18,60 & $3,00 \mid$ & 3,19 & 5,26 & 0,44 & $"$ & 21 & 87 \\
\hline 1. Februar & 2,01 & 22,80 & 3,80 & 4,26 & 7,02 & 0,49 & " & 26 & 108 \\
\hline & 1,34 & 29,20 & 5,00 & 7,09 & 11,69 & 0,48 & $n$ & 22 & 90 \\
\hline 15. & 1,63 & 32,20 & 6,10 & 7,80 & 12,86 & 0,50 & $n$ & 36 & 107 \\
\hline 22. $"$ & 2,07 & 28,40 & 4,20 & 5,67 & 9,35 & 0,58 & $n$ & 32 & 89 \\
\hline 1. März & 2,10 & 26,00 & $|4,20|$ & 5,32 & 8,77 & 0,48 & $n$ & 23 & 63 \\
\hline 8. " & 2,46 & 30,80 & 4,80 & 6,38 & 10,52 & 0,49 & $n$ & 22 & 50 \\
\hline 15. & 4,10 & 19,40 & 4,00 & 3,19 & 5,26 & 0,43 & $"$ & 66 & 147 \\
\hline 22. " & 3,98 & 19,00 & 3,00 & 2,84 & 4,68 & 0,36 & $n$ & 56 & 107 \\
\hline $29 . \quad "$ & 3,33 & 19,20 & $3,60 \mid$ & 3,19 & 5,26 & 0,48 & $"$ & 41 & 80 \\
\hline 6. April & 2,65 & 21,80 & 3,30 & 3,55 & 5,85 & 0,37 & $n$ & 22 & 64 \\
\hline 12. $"$ & 2,69 & 24,40 & 3,60 & 4,96 & 8,18 & 0,33 & $n$ & 39 & 90 \\
\hline 19. & 2,63 & 21,60 & $4,20 \mid$ & 3,55 & 5,85 & 0,35 & $"$ & 26 & 53 \\
\hline 26. . & 2,87 & 20,80 & $|3,40|$ & 4,61 & 7,60 & 0,36 & $"$ & 46 & 93 \\
\hline 3. Mai & 3,51 & 19,40 & $3,60 \mid$ & 3,55 & 5,85 & 0,37 & $n$ & - & 59 \\
\hline $10 . " 1$ & 2,14 & 22,00 & $4,60 \mid$ & 4,61 & 7,60 & 0,33 & $"$ & 63 & 137 \\
\hline 17. " & 1,62 & 25,40 & 5,50 & 6,03 & 9,94 & $\mid 0,39$ & $"$ & 58 & - \\
\hline 25. " & 1,63 & 32,00 & 5,10 & 8,87 & 14,62 & 0,42 & $n$ & - & - \\
\hline 31. " " & 1,12 & 29,30 & $|5,40|$ & 7,80 & 12,86 & 0,41 & $"$ & 101 & 248 \\
\hline 7. Juni & 0,88 & 39,60 & 4,60 & 12,41 & 20,46 & 0,58 & $n$ & 56 & 124 \\
\hline 14. $\quad "$ & 0,74 & 47,40 & $|7,00|$ & 15,60 & 25,74 & 0,53 & $n$ & 36 & 56 \\
\hline 20. & 0,59 & 52,60 & 6,60 & 17,73 & 29,26 & 0,54 & $" n$ & 42 & 78 \\
\hline 28. " & 0,40 & 60,20 & $|6,90|$ & 21,63 & $|35,66|$ & $|0,60|$ & $n$ & 18 & 26 \\
\hline 5. Juli & 0,54 & 65,60 & 8,00 & 23,40 & 38,58 & 0,54 & $"$ & 38 & 44 \\
\hline 12. $"$ & 0,70 & 35,60 & 6,80 & 10,99 & $18,12\}$ & 0,58 & $"$ & 16 & 32 \\
\hline 19. & 0,63 & 47,60 & 5,80 & 17,02 & 28,06 & 0,54 & $"$ & 16 & - \\
\hline 26. & 0,66 & 44,30 & 7,10 & 14,89 & $24,55 \mid$ & 0,73 & $n$ & 42 & 66 \\
\hline 2. August & 0,58 & 50,60 & 6,80 & 17,38 & 28,65 & $0,62 \mid$ & $n$ & - & - \\
\hline & 0,44 & 45,60 & 7,40 & 15,25 & 25,14 & 0,59 & $n$ & 34 & 98 \\
\hline 16. & 0,91 & 43,20 & 6,20 & 13,47 & $22,21\}$ & 0,57 & $"$ & 34 & 52 \\
\hline & 0,99 & 30,80 & $4,40 \mid$ & 7,80 & 12,86 & 0,74 & $n$ & 16 & - \\
\hline 30. " & 1,11 & 31,20 & 4,50 & 8,51 & 14,03 & 0,63 & $"$ & 24 & - \\
\hline 6. September & 0,76 & 35,80 & 5,40 & 10,64 & 17,56 & 0,66 & " & 12 & \\
\hline $13 . \quad "$ & 1,84 & 27,00 & 4,20 & 6,74 & 11,11 & $0,62 \mid$ & $n$ & 16 & \\
\hline 20. & 1,04 & 25,80 & $4,50 \mid$ & 7,45 & $12,28 \mid$ & 0,63 & $n$ & 22 & 68 \\
\hline & 0,83 & 29,60 & 5,00 & $9,22 !$ & 15,20 & 0,53 & $n$ & 22 & \\
\hline 4. Oktober & 0,90 & 32,40 & 5,40 & 9,57 & 15,78 & 0,67 & 1 & 6 & 10 \\
\hline 11. $"$ & 3,08 & 24,00 & 4,40 & 6,03 & 9,94 & 0,58 & $n$ & 24 & \\
\hline 18. & 4,02 & 16,40 & $3,60 \mid$ & 3,90 & 6,43 & 0,73 & $"$ & 10 & 22 \\
\hline 25. & 2,20 & 19,20 & $3,40 \mid$ & 3,90 & 6,43 & 0,49 & n & 14 & 44 \\
\hline 1. November & 1,50 & 25,40 & 3,60 & 6,38 & $|10,52|$ & $\mid 0,52$ & $n$ & 4 & 72 \\
\hline $8 . \quad$, & 1,55 & 29,60 & 4,70 & 7,80 & 12,86 & 0,49 & $" 1$ & 8 & \\
\hline 15. & 1,56 & 26,40 & $|4,60|$ & 6,38 & 10,52 & 0,49 & $n$ & 28 & 50 \\
\hline 22. & 1,48 & 29,60 & $5,00 \mid$ & 7,09 & 11,69 & 0,48 & $n$ & 14 & 58 \\
\hline & 1,26 & 30,80 & $4,70 \mid$ & 7,45 & 12,28 & 0,53 & $"$ & 24 & \\
\hline 6. Dezember & 1,71 & 35,40 & 5,40 & 8,87 & 14,62 & 0,49 & $n$ & 84 & 210 \\
\hline 13. & 3,43 & 23,00 & 4,80 & 4,26 & 7,02 & 0,47 & $n$ & 10 & 66 \\
\hline 20. & 3,30 & 18,80 & $3,60 \mid$ & 2,84 & 4,68 & 0,47 & $n$ & 30 & \\
\hline 27. & 2,20 & 21,20 & $3,80 \mid$ & 3,90 & 6,43 & 0,55 & $"$ & 6 & \\
\hline & & & 4,81 & 8,09 & 13,35 & 0,51 & fehlen & 31 & 82 \\
\hline
\end{tabular}

1) Angew. Chem. 25, 276-280, 1382[1912]; 26, I, 171-172 [1913]; 27, I, 119-120 [1914]; 28, I, 91-92 [1915].
Es folgen die monatlichen Durchschnittsanalysen von täglich entnommenen Proben, deren Untersuchung sich mit auf Schwefelsäure, Kalk und Magnesia unter Berechnung der letzteren beiden auf deutsche Härtegrade erstreckt. Dieser Durchschnitt muß, da er täglichen Probenahmen entspricht, eine durchaus zutreffende Wiedergabe von der durchschnittlichen Zusammensetzung des Magdeburger Leitungswassers (rechtes Elbufer) im Jahre 1915 ergeben. Tabelle II bringt die Resultate.

\section{Tabelle II.}

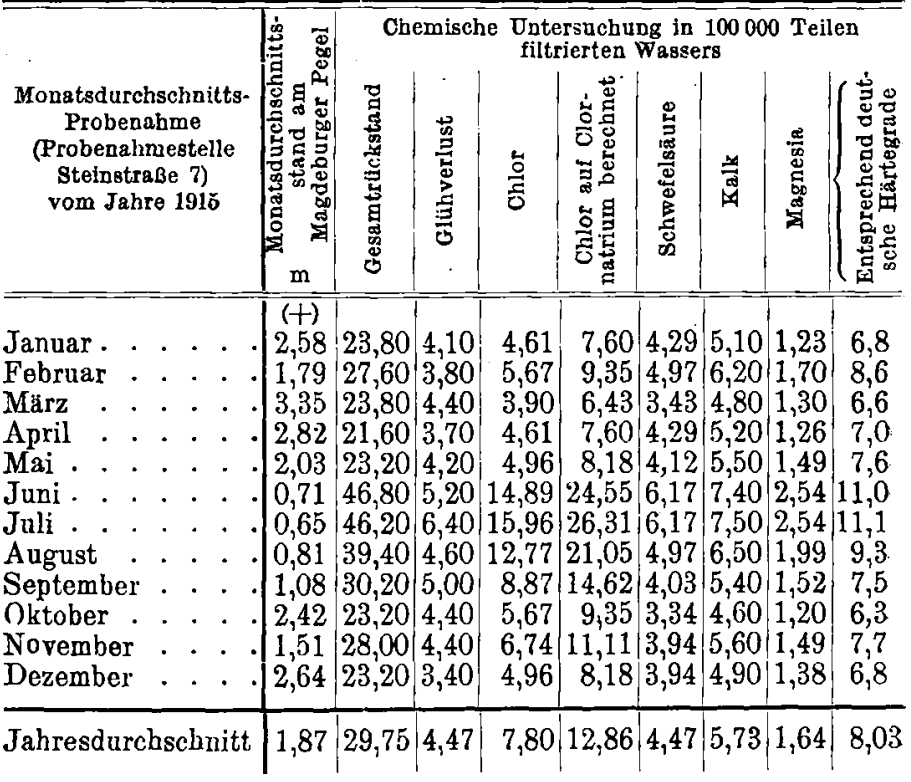

Es zeigt übrigens ein Blick auf die Tabellen I und II, daß die wöchentlichen und täglichen Probenahmeresultate nur wenig Unterschied ergeben. Tabelle I Tabelle II

$$
\begin{array}{cr}
\begin{array}{c}
\text { Tabelle I } \\
\text { wöchentliche } \\
\text { Probenahmen }
\end{array} & \begin{array}{c}
\text { Tabelle II } \\
\text { ttigliche } \\
\text { Probenahme } \\
+1,84
\end{array} \\
+\quad 1,87 \\
30,45 & 29,75 \\
4,81 & 4,47 \\
& \\
13,35 & 12,86
\end{array}
$$

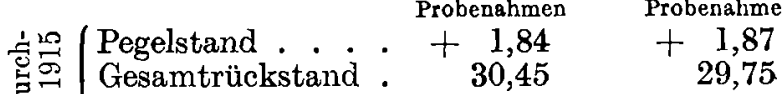$$
\text { 蛋 } \text { Glühverlust } \dot{0} \quad 4,81 \quad 4,47
$$$$
\text { Chlor, auf Chlor- }
$$

Die Durchschnittszahl für den Sauerstoffverbrauch (Oxydierbarkeit der organischen Substanz) beträgt nach Tabelle I, 0,51; die Durchschnittskeimzahlen nach 2 Tagen 31 , nach 5 Tagen 82.

War schon das Jahr 1914 mit einem Durchschnittspegelstand von $+1,34$ (bzw. $+1,35$ ) ein wasserreiches zu nennen, so übertraf der Wasserreichtum des Jahres 1915 mit einem Durchschnittspegelstand von $+1,84$ (bzw. $+1,87$ ) bei weitem den des vorhergehenden Jahres. Der niedrigste Wasserstand wurde festgestellt am 28.6 . mit $+0,40$ Pegel, der höchste am 14./3. mit $+4,63$ Pegel.

Von einer Tiefstandperiode kann im verflossenen Jahre überhaupt nicht die Rede sein. Wir haben nur in der abnorm heißen Juni/Juli-Zeit einen mäßig hohen Wasserstand zu verzeichnen, der sich bis zu Anfang September meist unter $+1 \mathrm{~m}$ Pegel hielt, mithin für unsere Magdeburger Trinkwasserverhältnisse immer noch im allgemeinen güstige Zahlenergebnisse aufwies.

Eine andauernde Hochwasserperiode fand statt vom Anfang Januar bis gegen. Ende Mai; ferner Anfang bis Ende Oktober und im Dezember.

Der Gehalt an gelösten Mineralbestandteilen des Magdeburger Trinkwassers ist bei 30,45, bzw. 29,75 Gesamtrückstand (Tabelle I und II) auch im vergangenen Jahre als recht günstig $\mathrm{zu}$ bezeichnen.

Ebenso günstig sind die Durchschnittskeimzahlen mit 31 nach zweitägiger Zählung bzw. 82 nach fünftägiger. Nur von wenigen Untersuchungen ergaben die Zählungen nach 5 Tagen etwas höhere Zahlen. Es haben mithin auch im vergangenen Jahre die Filteranlagen des Magdeburger Wasserwerkes vollauf befriedigend ihre Aufgabe erfüllt.

Für den Härtegrad des Wassers ist das gleiche zu sagen wie für den Gehalt an Mineralbestandteilen. Gesamtdurch- 
Tabelle III.

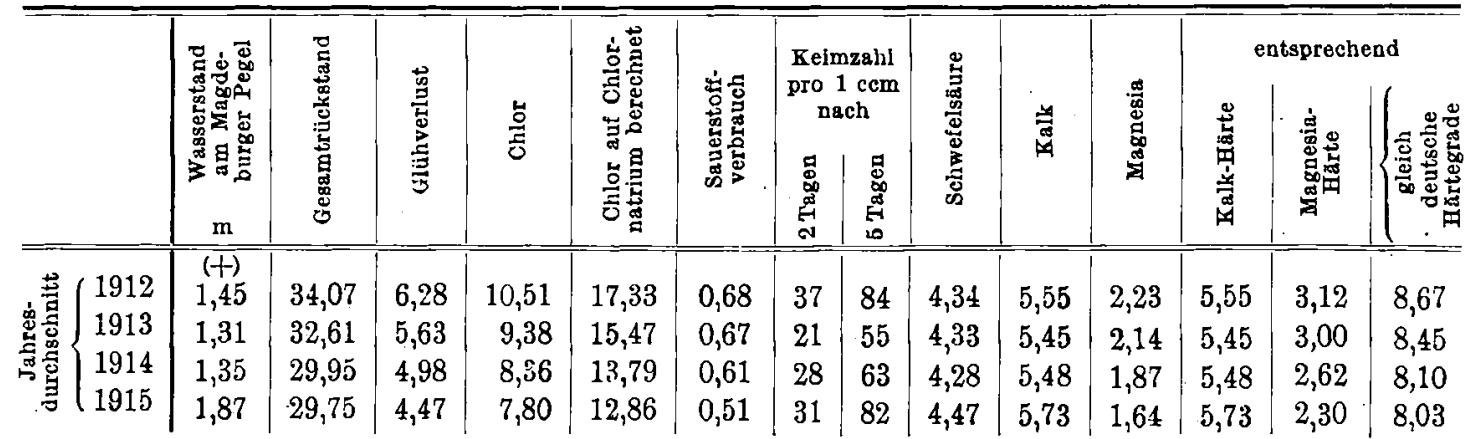

schnittshärtegrad des ganzen Jahres 1915 beträgt 8,03. Der niedrigste Härtegrad von 6,6 fällt auf den Monat März mit seinem. Höchstmonatsdurchschnittspegel von $+3,35$, der höchste Härtegrad von 11,1 fällt auf den Juli mit dem Niedrigstmonatsdurchschnittspegel von $+0,65$.

Tabelle III gibt eine übersichtliche Zusammenstellung der Jahresdurchschnitte mit Angabe von Schwefelsäure, Kalk und Magnesia aus den Jahren 1912, 1913, 1914, 1915.

Der Pegelstand des Jahres 1915 übertrifft nicht nur den des Jahres 1914, sondern auch die von 1912 und 1913. Es wäre dementsprechend zu erwarten, daß auch der Gesamtrückstand bzw. der Gehalt an gelösten Mineralbestandteilen im Jahre 1915 zurückgegangen sei. Es trifft diese Annahme zu fast gleichmäßig für beide Kriegsjahre; jedoch tritt sie lediglich in Erscheinung bei dem Gehalt an Chlor und an Magnesia, während der Gehalt an Schwefelsäure und Kalk sich, wie in den beiden Vorjahren 1912 und 1913, als ungefäbr gleichbleibende Dauerbestandteile der Elbe erwiesen. Dieser Rückgang an Chlor mit 8,36 und 7,80 in den Jahren 1914 und 1915 gegen 10,51 und 9,38 in den Jahren 1912 und 1913, sowie an Magnesia mit 1,87 und 1,64 in den Jahren 1914 und 1915 gegen 2,23 und 2,14 in 1912 und 1913 ist zurückzuführen auf die Minderverarbeitung an Kalisalzen in den letzten beiden Kriegsjahren.

Unliebsame Störungen durch Eisstand auf der Elbe waren auch im Jahre 1915 nicht zu verzeichnen.

Im ganzen Jahre 1915 haben wir - abgesehen von gelöster organischer Substanz, deren Vorhandensein ich in allen meinen Veröffentlichungen besondere Aufmerksamkeit widmete - wiederum ein gutes Trinkwasser zur Verfügung gehabt.

Die niedrigste Sauerstoffzahl von 0,33 zeigt die Untersuchung vom 12./4. bei $+2,69$ Pegel, die höchste von 0,74 die vom 23./8. mit $+0,99$ Pegel. Der Jahresdurchschnitt mit 0,51 ist immerhin günstiger als der des vergangenen Jahres mit 0,61 .

Seit 3 Jahren haben wir unsere Untersuchungen des Magdeburger Leitungswassers folgend erweitert:

Von der Gesamtmagnesia bzw. Gesamtmagnesiahärte ist analytisch noch festgestellt worden, wieviel davon auf Carbonathärte und wieviel auf bleibende Härte entfällt. Ich nehme dabei im allgemeinen an, daß die Carbonathärte einen Ausdruck gibt für die Magnesia, die aus Dolomit oder magnesiumhaltigen Silicatgesteinen in das Elbwasser gelangt, während die bleibende Härte die Magnesia anzeigt, die durch die Abwässer der Kaliindustrie als Chlormagnesium dem Flusse zugeführt wird.

Behufs Feststellung dieser beiden Magnesiaarten haben wir uns der von Professor H. P. re ch t ausgearbeiteten Alkoholauswaschmethode bedient, welche zu relativem Vergleich hinlänglich genaue Resultate ergibt²).

Zur Untersuchung wurde jedesmal die für Tabelle II zur Unterlage dienende Monatsdurchschnittsprobe, hergestellt aus täglichen Probenahmen, verwendet, so daß also auch hier ein absolut zuverlässiger Durchschnitt aus täglichen, während des ganzen Jahres entnommenen Proben vorliegt.

Die Befunde der Monatsdurchschnitte betreffender 3. Jahre zeigt Tabelle IV.

Aus der Zusammenstellung der Jahresdurchschnitte dieser Tabelle ist zu ersehen, einmal, daß während der 3 Untersuchungsjahre - mit ihrem befriedigend hohen

2) Vgl. auch $P$ f e if f e $r$, Angew. Chem. 29, I, 7 [1916].
Pegelstande - namentlich im zweiten Kriegsjahr (im ersten Kriegsjahr kommen nur 5 Monate in Betracht) weniger Magnesia eingeführt wurde als im Friedensjahr 1913 und

Tabelle IV

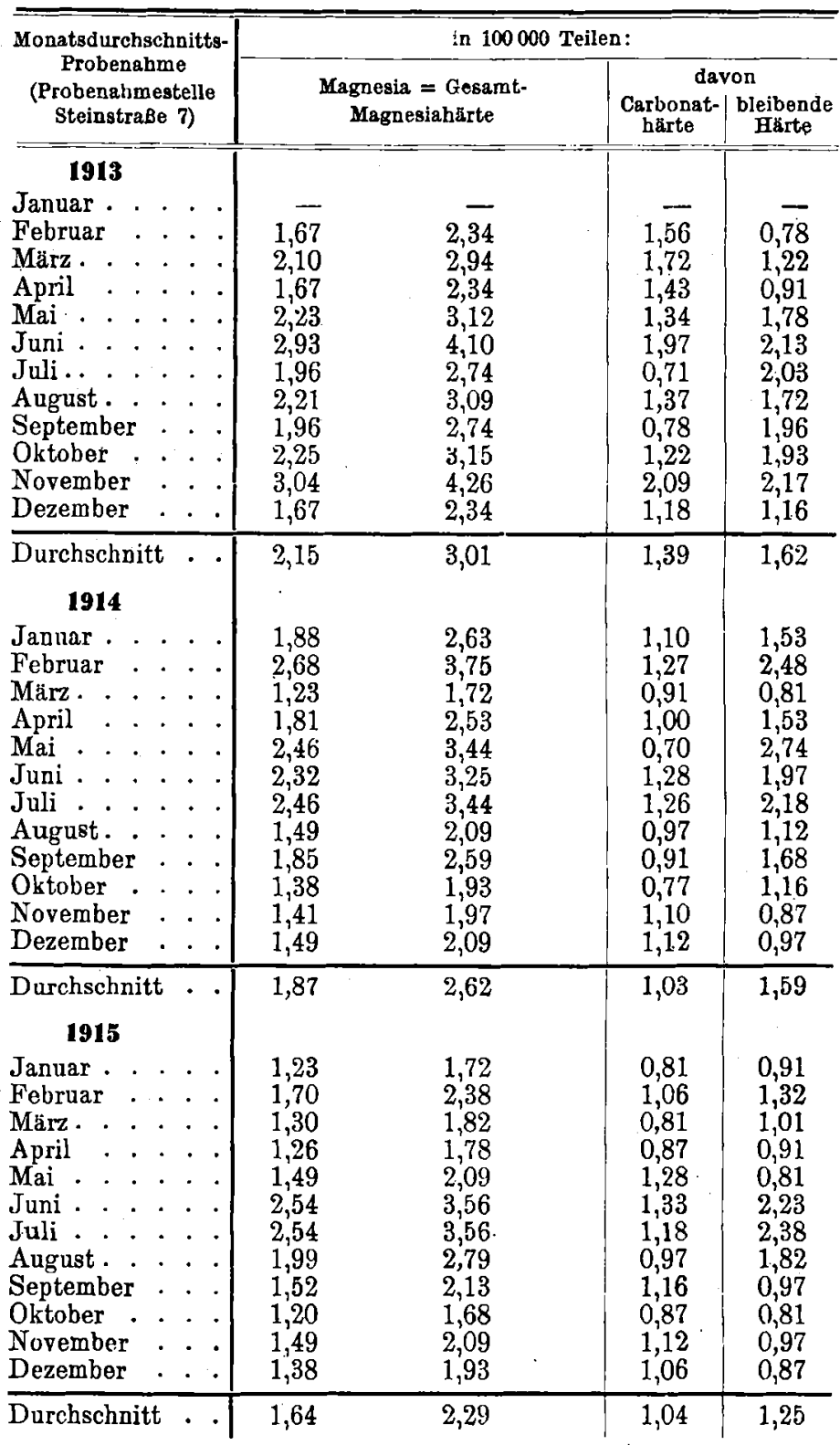

ferner, daß die Zahlen für die bleibende Härte ü ber $h$ a u pt nur geringfügig sind, $d . h$., daß die Menge der durch die Kaliindustrie unserem Trinkwasser zugeführten Magnesia unwesentlich ins Gewicht fällt!

[A. 14.]

Berichtigung: In dem Aufsatz von K. v. Allwörden : Die Eigenschaften der Schafwolle und eine neue Untersuchungsmethode zum Nachweis geschädigter Wolle auf chemischem Wege (Angew. Chem. 29, I, 77 [1916]) muß es Zeile 17 von vorne heißen: ..., der in Alkalien leicht löslich ist, ... 